\title{
Une caractérisation des rétractes absolus de voisinage
}

\author{
par \\ Robert Cauty (Paris)
}

\begin{abstract}
We prove that a metric space is an ANR if, and only if, every open subset of $X$ has the homotopy type of a CW-complex.
\end{abstract}

1. Introduction. Le but de cet article est de prouver le résultat suivant.

ThÉORÈme. Un espace métrisable $X$ est un rétracte absolu de voisinage si, et seulement si, tout ouvert de $X$ a le type d'homotopie d'un $C W$ complexe.

La nécessité est évidente puisque tout ouvert d'un rétracte absolu de voisinage en est encore un et que tout rétracte absolu de voisinage a le type d'homotopie d'un CW-complexe. Le problème de savoir si la réciproque est vraie a été posé par R. Geoghegan [6] et figure dans sa liste classique de problèmes sur la topologie de la dimension infinie [7] sous la référence ANR2, ainsi que dans la nouvelle liste de J. West [14] (mais rebaptisé là ANR3).

L'outil principal de la démonstration est la réalisation géométrique $S(X)$ du complexe singulier de $X$. L'application naturelle $\pi$ de $S(X)$ dans $X$ est une équivalence homotopique faible, donc une équivalence homotopique lorsque $X$ a le type d'homotopie d'un CW-complexe. Pour tout sous-ensemble $U$ de $X, S(U)$ s'identifie à un sous-complexe de $S(X)$, donc, dans la situation du théorème, la restriction de $\pi$ à $S(U)$ est, pour tout ouvert $U$, une équivalence homotopique de $S(U)$ dans $U$. Cette propriété nous permettra de construire une fonction continue $\varphi: X \times] 0,1] \rightarrow S(X)$ et une homotopie $\Phi: X \times[0,1] \rightarrow X$ vérifiant $\Phi(x, 0)=x$ et $\Phi(x, t)=\pi \circ \varphi(x, t)$ pour $t>0$. L'existence de ces fonctions entraîne que, pour tout recouvrement ouvert $\mathfrak{U}$ de $X, X$ est $\mathfrak{U}$-dominé par $S(X)$ (si la fonction $\lambda: X \rightarrow] 0,1]$ est telle que, pour tout $x \in X, \Phi(\{x\} \times[0, \lambda(x)])$ soit contenu dans un élément de $\mathfrak{U}$, et si $f: X \rightarrow S(X)$ est définie par $f(x)=\varphi(x, \lambda(x))$, alors $\pi \circ f$ est $\mathfrak{U}$ -

1991 Mathematics Subject Classification: Primary 54C55. 
homotope à l'identité de $X$ ). Comme $S(X)$ est un CW-complexe (et même triangulable), un théorème de Hanner ([9], théorème 6.3 , p. 139) entraîne alors que $X$ est un rétracte absolu de voisinage. La construction de $\varphi$ et $\Phi$ utilise le cylindre de l'application $\pi$ d'une façon analogue à celle utilisée dans [10] par G. Kozlowski, mais la technique est ici beaucoup plus complexe que dans [10] car l'application $\pi$ n'est pas fermée et $S(U)$, qui jouera le rôle joué par $\pi^{-1}(U)$ dans [10], est en général un sous-ensemble propre de $\pi^{-1}(U)$ dont l'intérieur dans $S(X)$ est vide.

La distance sur un espace métrique sera toujours notée $d ; \operatorname{diam}(U)$ désignera le diamètre d'un sous-ensemble $U$ de $X$. Si $\mathfrak{U}=\left\{U_{\alpha} \mid \alpha \in A\right\}$ est une famille de sous-ensembles de $X$, nous noterons $\overline{\mathfrak{U}}=\left\{\bar{U}_{\alpha} \mid \alpha \in A\right\}$ la famille des fermetures des éléments de $\mathfrak{U}$ et $N(\mathfrak{U})$ le nerf de la famille $\mathfrak{U} ;\left\langle\alpha_{0}, \ldots, \alpha_{n}\right\rangle$ désignera le simplexe de $N(\mathfrak{U})$ correspondant aux sommets $U_{\alpha_{0}}, \ldots, U_{\alpha_{n}}$. Nous poserons $I=[0,1]$. Une fonction $f: X \rightarrow Y$ sera dite inessentielle si elle est homotope à une fonction constante.

2. Préliminaires. Pour tout espace $X$, nous noterons $S(X)$ la réalisation géométrique du complexe singulier de $X$ et $\pi_{X}$ - ou simplement $\pi$ l'application naturelle de $S(X)$ dans $X$. Nous utiliserons la construction de $S(X)$ donnée par J. B. Giever [8] (qui le note $\bar{P}(X)$ ), qui ne tient pas compte des dégénérescences. Pour $U \subset X$, nous identifions $S(U)$ à un souscomplexe de $S(X)$ et, si $\mathfrak{U}=\left\{U_{\alpha} \mid \alpha \in A\right\}$ est une famille de sous-ensembles de $X$, nous posons $S(\mathfrak{U})=\bigcup_{\alpha \in A} S\left(U_{\alpha}\right) ; S(\mathfrak{U})$ est un sous-complexe de $S(X)$. Il est clair que si $U$ et $V$ sont deux sous-ensembles de $X$, alors $S(U) \cap S(V)=S(U \cap V)$.

LEMme 1. Si $\mathfrak{U}$ est une famille de sous-ensembles de $X$ dont les intérieurs recouvrent $X$, alors l'inclusion $i: S(\mathfrak{U}) \hookrightarrow S(X)$ est une équivalence homotopique.

Démonstration. Il suffit de montrer que $i$ est une équivalence homotopique faible. Puisque $\pi \mid S(\mathfrak{U})=\pi \circ i$ et que $\pi$ est une équivalence homotopique faible ([8], théorème VI), il suffit de montrer que $\pi \mid S(\mathfrak{U})$ en est une, mais cela est implicite dans les arguments des sections 8 et 9 de [8] (voir, en particulier, le lemme 2, page 187).

Etant donnée une fonction continue $f: X \rightarrow Y$, le cylindre $M(f)$ de $f$ est l'espace quotient de la somme topologique $X \times I \amalg Y$ obtenu en identifiant $(x, 0)$ à $f(x)$ pour tout $x \in X$. Soit $q$ la projection naturelle de $X \times I \amalg Y$ sur $M(f)$. Nous poserons $[x, t]=q(x, t)$ pour tout $(x, t) \in X \times I$, et nous identifierons naturellement $X$ et $Y$ aux sous-espaces $q(X \times\{1\})$ et $q(Y)$ respectivement de $M(f)$.

Rappelons qu'un sous-espace $A$ d'un espace $X$ est appelé un rétracte par déformation forte de $X$ s'il existe une homotopie $k: X \times I \rightarrow X$ vérifiant 
$k(x, t)=x$ pour $(x, t) \in(X \times\{0\}) \cup(A \times I)$ et $k(X \times\{1\}) \subset A$; une telle homotopie est appelée une rétraction par déformation forte de $X$ sur $A$.

Lemme 2. Si $f: X \rightarrow Y$ est une équivalence homotopique, alors $X$ est un rétracte par déformation forte de $M(f)$.

Ce lemme résulte immédiatement des théorèmes 1.26 et 2.29 de [3].

Pour tout espace $X$, nous poserons $M(X)=M\left(\pi_{X}\right)$. Si $U$ est un sousespace de $X, \pi_{U}(a)=\pi_{X}(a)$ pour tout $a \in S(U)$, donc $M(U)$ s'identifie naturellement à un sous-ensemble de $M(X)$. La remarque élémentaire suivante sera utilisée implicitement dans toute la suite.

Lemme 3. Pour tout sous-espace $U$ de $X$, la topologie induite par $M(X)$ sur $M(U)$ coïncide avec la topologie du cylindre de l'application $\pi_{U}$.

Démonstration. Soient $q: S(X) \times I \amalg X \rightarrow M(X)$ et $q^{\prime}: S(U) \times$ $I \amalg U \rightarrow M(U)$ les projections naturelles. La restriction de $q$ à $S(U) \times I \amalg U$ étant continue, la topologie $\mathfrak{T}$ induite par $M(X)$ sur $M(U)$ est moins fine que la topologie $\mathfrak{T}^{\prime}$ du cylindre de $\pi_{U}$. Inversement, soit $F \subset M(U)$ fermé pour $\mathfrak{T}^{\prime}$; alors $q^{\prime-1}(F)$ est fermé dans $S(U) \times I \amalg U$ et $F \cap U$ est fermé dans $U$. Soit $G$ la fermeture de $F \cap U$ dans $X$, et soit $H=\left(q^{\prime-1}(F) \cap\right.$ $(S(U) \times I)) \cup\left(\left(\pi_{X}^{-1}(G) \times\{0\}\right) \cup G\right)$. Puisque $S(U)$, étant un sous-complexe, est fermé dans $S(X), q^{\prime-1}(F) \cap(S(U) \times I)$ est fermé dans $S(X) \times I$, donc $H$ est fermé dans $S(X) \times I \amalg X$. On vérifie facilement que $H$ est saturé pour la relation d'équivalence définissant $M(X)$, donc $q(H)$ est fermé dans $M(X)$, et que $q(H) \cap M(U)=F$, ce qui montre que $H$ est fermé pour $\mathfrak{T}$, donc que $\mathfrak{T}^{\prime}$ est moins fine que $\mathfrak{T}$, d'où l'égalité.

Pour tout sous-ensemble $U$ de $X, \overline{M(U)} \backslash M(U) \subset X$ et $M(U)$ est fermé si, et seulement si, $U$ est fermé. Le fait que $S(U) \cap S(V)=S(U \cap V)$ entraîne

$$
M(U) \cap M(V)=M(U \cap V)
$$

pour tous les sous-ensembles $U$ et $V$ de $X$. Si $\mathfrak{U}=\left\{U_{\alpha} \mid \alpha \in A\right\}$ est une famille de sous-ensembles de $X$, nous poserons $M(\mathfrak{U})=\bigcup_{\alpha \in A} M\left(U_{\alpha}\right)$, que nous regardons comme un sous-espace de $M(X)$; si $\mathfrak{U}$ recouvre $X$, alors $M(\mathfrak{U})$ contient $X$. Nous noterons $\varrho$ la rétraction de $M(X)$ sur $X$ définie par $\varrho(x)=x$ si $x \in X$ et $\varrho([a, t])=\pi(a)$ pour $(a, t) \in S(X) \times I$.

Lemme 4. Si $\mathfrak{U}$ est une famille de sous-ensembles d'un espace $X$ dont les intérieurs recouvrent $X$, alors $S(X) \cup M(\mathfrak{U})$ est un rétracte par déformation forte de $M(X)$.

Démonstration. Puisque $S(\mathfrak{U})$ est un sous-complexe de $S(X)$, l'inclusion $S(\mathfrak{U}) \hookrightarrow S(X)$ est une cofibration; d'après le lemme 1, c'est une équivalence homotopique. D'après le théorème 6 de [13], l'inclusion de $(S(X)$ $\times\{0,1\}) \cup(S(\mathfrak{U}) \times I)$ dans $S(X) \times I$ est une cofibration et une équivalence homotopique. D'après le théorème 2.29 de $[3],(S(X) \times\{0,1\}) \cup(S(\mathfrak{U}) \times I)$ 
est un rétracte par déformation forte de $S(X) \times I$, donc $(S(X) \times\{0,1\}) \cup$ $(S(\mathfrak{U}) \times I) \amalg X$ est un rétracte par déformation forte de $S(X) \times I \amalg X$. Le lemme s'en déduit par passage au quotient.

Lemme 5. Soit $\mathfrak{U}$ une famille de sous-ensembles d'un espace $X$ dont les intérieurs recouvrent $X$. Alors, $((S(X) \cup M(\mathfrak{U})) \times I) \cup(M(X) \times\{0,1\})$ est un rétracte par déformation forte de $M(X) \times I$.

Démonstration. Si $\omega: S(X) \rightarrow I$ vérifie $\omega^{-1}(0)=S(\mathfrak{U})$, alors la fonction $\alpha: M(X) \rightarrow I$ définie par $\alpha(x)=0$ si $x \in X$ et $\alpha([a, t])=$ $\min (t(1-t), \omega(a))$ pour $(a, t) \in S(X) \times I$ vérifie $\alpha^{-1}(0)=S(X) \cup M(\mathfrak{U})$. Combinant les théorèmes 2.29 et 3.26 de [3] avec le lemme 4 , nous constatons que l'inclusion de $S(X) \cup M(\mathfrak{U})$ dans $M(X)$ est une cofibration et une équivalence homotopique. Le théorème 6 de $[13]$ garantit alors qu'il en est de même de l'inclusion de $((S(X) \cup M(\mathfrak{U})) \times I) \cup(M(X) \times\{0,1\})$ dans $M(X) \times I$, d'où le résultat ([3], théorème 2.29$)$.

Rappelons qu'un espace topologique $X$ est dit stratifiable [1] s'il est séparé et s'il existe une fonction associant à tout ouvert $U$ de $X$ une suite d'ouverts $\left\{U_{n}\right\}_{n=1}^{\infty}$ de façon que (a) $\bar{U}_{n} \subset U$, (b) $U=\bigcup_{n=1}^{\infty} U_{n}$ et (c) $U \subset V$ implique $U_{n} \subset V_{n}$ pour tout $n$. Il est connu (voir [1]) que tout espace stratifiable est paracompact, que tout espace métrisable est stratifiable, ainsi que tout CW-complexe, tout sous-espace d'un espace stratifiable et tout produit dénombrable de tels espaces.

Lemme 6. Soit $f: X \rightarrow Y$ une fonction continue. Si $X$ et $Y$ sont stratifiables, $M(f)$ aussi.

C'est un cas particulier du théorème 6.2 de [1].

Ce lemme entraîne en particulier que $M(X)$ est stratifiable lorsque $X$ est métrisable; c'est le cas dont nous aurons besoin dans la suite.

Un espace $X$ est appelé un rétracte absolu de voisinage pour la classe des espaces stratifiables - ou RAV(stratifiable) — s'il est stratifiable et si, pour tout fermé $A$ d'un espace stratifiable $Y$, toute fonction continue de $A$ dans $X$ se prolonge à un voisinage de $A$ dans $Y$. Le théorème d'extension des homotopies de Borsuk s'étend comme suit aux espaces stratifiables.

Lemme 7. Soient $X$ un $R A V$ (stratifiable), $Y$ un espace stratifiable et $A$ un fermé de $Y$. Etant données une fonction continue $f: Y \rightarrow X$ et une homotopie $h: A \times I \rightarrow X$ vérifiant $h(a, 0)=f(a) \forall a \in A$, il existe une homotopie $H: Y \times I \rightarrow X$ telle que $H(x, 0)=f(x) \forall x \in Y$ et $H \mid A \times I=h$.

Dém on stration. Puisque $Y \times I$ est stratifiable, donc normal, cela est une conséquence immédiate du lemme 2.1, page 116, de [9].

Enfin, le lemme suivant est un cas particulier du corollaire 2.4 de [2].

Lemme 8. Tout $C W$-complexe est un RAV(stratifiable). 
3. Deux résultats auxiliaires. Dans toute cette section, $X$ est un espace métrisable dont tout ouvert a le type d'homotopie d'un CW-complexe. Alors, pour tout ouvert $U, \pi \mid S(U)$ est une équivalence homotopique de $S(U)$ dans $U$ et, d'après le lemme $2, S(U)$ est un rétracte par déformation forte de $M(U)$.

Lemme 9. Soient $A$ un fermé de $X, Q$ un voisinage ouvert de $A$ dans $X$ et $K$ un $R A V$ (stratifiable). Si $f$ est une fonction continue de $S(X) \cup M(Q)$ dans $K$, alors il existe une fonction continue $g$ de $M(X)$ dans $K$ telle que $g|S(X) \cup M(A)=f| S(X) \cup M(A)$.

Démonstration. Puisque $X$ a le type d'homotopie d'un CW-complexe, il admet un recouvrement ouvert $\mathfrak{P}$ tel que, pour tout $P \in \mathfrak{P}$, l'inclusion $P \hookrightarrow X$ soit inessentielle (voir, par exemple, [3], lemme 8, p. 235). Soit $\mathfrak{V}^{\prime}$ un recouvrement ouvert localement fini et $\sigma$-discret de $X$ plus fin que $\mathfrak{P}$ et que le recouvrement $\{Q, X \backslash A\}$. Soit $V$ la réunion des éléments de $\mathfrak{V}^{\prime}$ contenus dans $Q$, et soit $\mathfrak{V}=\left\{V_{i} \mid i \in J\right\}$ la collection des éléments de $\mathfrak{V}^{\prime}$ qui ne sont pas contenus dans $Q$. Alors, $\{V\} \cup \mathfrak{V}$ est un recouvrement ouvert de $X$, donc nous pouvons trouver des ouverts $U$ et $U_{i}, i \in J$, avec $\bar{U} \subset V$ et $\bar{U}_{i} \subset V_{i}$ tels que $\mathfrak{U}=\{U\} \cup\left\{U_{i} \mid i \in J\right\}$ recouvre $X$. Puisque les éléments de $\mathfrak{V}$ sont contenus dans $X \backslash A, U$ contient $A$.

Comme $\mathfrak{V}^{\prime}$ est $\sigma$-discret, il en est de même de $\mathfrak{V}$; soit $\mathfrak{V}=\bigcup_{n=1}^{\infty} \mathfrak{V}_{n}$, où chaque $\mathfrak{V}_{n}=\left\{V_{i} \mid i \in J_{n}\right\}$ est une famille discrète. Nous pouvons supposer que $\left\{J_{n}\right\}_{n=1}^{\infty}$ est une partition de $J$. Pour $p \geq 0$, prenons des ouverts $U^{p}$ et $U_{i}^{p}, i \in J$, vérifiant, pour tout $p \geq 0$,

$$
\begin{gathered}
\bar{U} \subset U^{p+1} \subset \bar{U}^{p+1} \subset U^{p} \subset \bar{U}^{p} \subset V, \\
\bar{U}_{i} \subset U_{i}^{p+1} \subset \bar{U}_{i}^{p+1} \subset U_{i}^{p} \subset \bar{U}_{i}^{p} \subset V_{i} .
\end{gathered}
$$

Pour $n \geq 1$ et $p \geq 0$, soit $\mathfrak{U}_{n}^{p}=\left\{U^{p}\right\} \cup\left\{U_{i}^{p} \mid i \in J_{1} \cup \ldots \cup J_{n}\right\}$. Soit $\mathfrak{U}_{0}^{0}=\left\{U^{0}\right\}$.

Affirmation 1. Pour $n \geq 0$, il existe des fonctions continues $h_{n}$ : $S(X) \cup M\left(\overline{\mathfrak{U}}_{n}^{n}\right) \rightarrow K$ vérifiant

$$
\begin{aligned}
h_{0} & =f \mid S(X) \cup M\left(\overline{\mathfrak{U}}_{0}^{0}\right), \\
h_{n} \mid S(X) \cup M\left(\overline{\mathfrak{U}}_{n-1}^{n}\right) & =h_{n-1} \mid S(X) \cup M\left(\overline{\mathfrak{U}}_{n-1}^{n}\right) \quad \text { pour } n \geq 1 .
\end{aligned}
$$

Supposons cette affirmation démontrée. En raison de (1)-(4), nous pouvons alors définir une fonction $h: S(X) \cup M(\overline{\mathfrak{U}}) \rightarrow K$ par

$$
\begin{gathered}
h|S(X) \cup M(\bar{U})=f| S(X) \cup M(\bar{U}), \\
h\left|M\left(\bar{U}_{i}\right)=h_{n}\right| M\left(\bar{U}_{i}\right) \quad \text { si } i \in J_{n} .
\end{gathered}
$$

Pour voir que $h$ est continue, remarquons d'abord que la finitude locale de la famille $\overline{\mathfrak{U}}$ dans $X$ entraîne que la famille $\left\{M\left(\bar{U}_{i}\right) \mid i \in J\right\}$ est localement finie dans $M(X)$. Comme la restriction de $h$ à chacun des ensembles $S(X) \cup$ 
$M(\bar{U})$ et $M\left(\bar{U}_{i}\right), i \in J$, qui sont fermés dans $M(X)$ et forment une famille localement finie, est continue, $h$ est continue. Le lemme 4 entraîne l'existence d'une fonction $g: M(X) \rightarrow K$ qui prolonge $h$; puisque $U$ contient $A$, c'est la fonction cherchée.

La condition (3) définit $h_{0}$. Soit $n \geq 1$ et supposons $h_{n-1}$ construite.

Affirmation 2. Pour tout $i \in J_{n}$, il existe une fonction continue $h_{i}$ : $M\left(U_{i}^{n-1}\right) \rightarrow K$ coïncidant avec $h_{n-1}$ sur $M\left(U_{i}^{n-1}\right) \cap\left(S(X) \cup M\left(\overline{\mathfrak{U}}_{n-1}^{n}\right)\right)$.

Notons d'abord que l'affirmation 2 entraîne l'affirmation 1 car, les éléments de $\mathfrak{V}_{n}$ étant deux à deux disjoints, elle nous permet de définir $h_{n}$ par

$$
\begin{gathered}
h_{n}\left|S(X) \cup M\left(\overline{\mathfrak{U}}_{n-1}^{n}\right)=h_{n-1}\right| S(X) \cup M\left(\overline{\mathfrak{U}}_{n-1}^{n}\right), \\
h_{n}\left|M\left(\bar{U}_{i}^{n}\right)=h_{i}\right| M\left(\bar{U}_{i}^{n}\right) \quad \text { pour } i \in J_{n} .
\end{gathered}
$$

La continuité de $h_{n}$ se vérifie comme celle de $h$ ci-dessus.

Pour $i$ dans $J_{n}$, soit $\Re_{i}$ la famille des ouverts $U_{i}^{n-1} \cap U^{n-1}$ et $U_{i}^{n-1} \cap$ $U_{j}^{n-1}, j \in J_{1} \cup \ldots \cup J_{n-1}$; soit $R_{i}$ la réunion des éléments de $\Re_{i}$. Le lemme 4 garantit l'existence d'une fonction continue $k_{i}: M\left(R_{i}\right) \rightarrow K$ coïncidant avec $h_{n-1}$ sur $M\left(\Re_{i}\right) \cup S\left(R_{i}\right)$. Alors, (4) permet de définir une fonction $\bar{k}_{i}$ : $M\left(R_{i}\right) \cup S\left(U_{i}^{n-1}\right) \rightarrow K \operatorname{par} \bar{k}_{i} \mid M\left(R_{i}\right)=k_{i}$ et $\bar{k}_{i}\left|S\left(U_{i}^{n-1}\right)=h_{n-1}\right| S\left(U_{i}^{n-1}\right)$; cette fonction est continue puisque ses restrictions à $M\left(R_{i}\right)$ et $S\left(U_{i}^{n-1}\right)$ le sont et que ces ensembles sont fermés dans $M\left(R_{i}\right) \cup S\left(U_{i}^{n-1}\right)$.

AfFirmation 3. $\bar{k}_{i}$ est inessentielle.

L'affirmation 3 entraîne l'affirmation 2. En effet, puisque $M\left(U_{i}^{n-1}\right) \cap$ $S(X)=S\left(U_{i}^{n-1}\right)$ et que $M\left(U_{i}^{n-1}\right) \cap M\left(\overline{\mathfrak{U}}_{n-1}^{n}\right) \subset M\left(U_{i}^{n-1}\right) \cap M\left(\Re_{i}\right)$, la définition de $\bar{k}_{i}$ et l'affirmation 3 entraînent que la restriction de $h_{n-1}$ au sous-ensemble $M\left(U_{i}^{n-1}\right) \cap\left(S(X) \cup M\left(\overline{\mathfrak{U}}_{n-1}^{n}\right)\right)$, qui est fermé dans $M\left(U_{i}^{n-1}\right)$, est inessentielle, donc, à l'aide du lemme 7 , se prolonge en une fonction continue $h_{i}: M\left(U_{i}^{n-1}\right) \rightarrow K$.

Pour prouver l'affirmation 3, considérons d'abord le diagramme commutatif

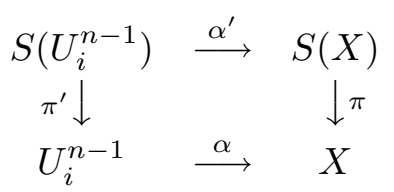

où $\alpha, \alpha^{\prime}$ sont des inclusions et $\pi^{\prime}$ la restriction de $\pi$. Puisque $U_{i}^{n-1}$ est contenu dans un élément de $\mathfrak{P}, \alpha$ est inessentielle. Puisque $X$ et $U_{i}^{n-1}$ sont ouverts, $\pi$ et $\pi^{\prime}$ sont des équivalences homotopiques. Par suite, $\alpha^{\prime}$ est inessentielle; comme $h_{n-1}$ est définie sur $S(X), h_{n-1} \mid S\left(U_{i}^{n-1}\right)$ est donc aussi inessentielle. 
Puisque $R_{i}$ est ouvert, $S\left(R_{i}\right)$ est un rétracte par déformation forte de $M\left(R_{i}\right)$. Comme $S\left(U_{i}^{n-1}\right) \cap M\left(R_{i}\right)=S\left(R_{i}\right), S\left(U_{i}^{n-1}\right)$ est donc un rétracte par déformation forte de $S\left(U_{i}^{n-1}\right) \cup M\left(R_{i}\right)$. Par suite, $\bar{k}_{i}$ est homotope à une fonction de la forme $\left(\bar{k}_{i} \mid S\left(U_{i}^{n-1}\right)\right) \circ r$, où $r$ est une rétraction de $S\left(U_{i}^{n-1}\right) \cup M\left(R_{i}\right)$ sur $S\left(U_{i}^{n-1}\right)$. Comme $\bar{k}_{i}\left|S\left(U_{i}^{n-1}\right)=h_{n-1}\right| S\left(U_{i}^{n-1}\right)$, cela entraîne l'inessentialité de $\bar{k}_{i}$, d'où le lemme.

Lemme 10. Soient $A$ un fermé de $X, Q$ un voisinage ouvert de $A$ dans $X$ et $K$ un $R A V$ (stratifiable). Si $F$ est une fonction continue de $((S(X) \cup$ $M(Q)) \times I) \cup(M(X) \times\{0,1\})$ dans $K$, alors il existe une fonction continue $G$ de $M(X) \times I$ dans $K$ qui coïncide avec $F$ sur $((S(X) \cup M(A)) \times I) \cup$ $(M(X) \times\{0,1\})$.

Démonstration. Prenons des ouverts $V, W$ vérifiant $A \subset V \subset \bar{V} \subset$ $W \subset \bar{W} \subset Q$. Soit $\mathfrak{U}=\{\bar{W}, X \backslash V\}$; les intérieurs des éléments de $\mathfrak{U}$ recouvrent $X$.

Affirmation. Il existe une fonction continue $H:(S(X) \cup M(\mathfrak{U})) \times I$ $\times I \rightarrow M(X) \times I$ vérifiant

(i) $H(a, t, s)=(a, t)$ si $(a, t, s) \in(S(X) \times I \times I) \cup(M(\mathfrak{U}) \times I \times\{0\})$,

(ii) $H(M(\mathfrak{U}) \times\{t\} \times I) \subset M(X) \times\{t\} \quad \forall t \in[0,1]$,

(iii) $H(M(A) \times I \times I) \subset M(Q) \times I$,

(iv) $H(M(\mathfrak{U}) \times I \times\{1\}) \subset S(X) \times I$.

Pour prouver cela, soit $\lambda: X \rightarrow I$ une fonction continue telle que $\lambda^{-1}(1)=A$ et $\lambda^{-1}(0)=X \backslash V$. Soit $k: M(Q) \times I \rightarrow M(Q)$ une rétraction par déformation forte de $M(Q)$ sur $S(Q)$, et soit $k^{\prime}: M(X) \times I \rightarrow M(X)$ une rétraction par déformation forte de $M(X)$ sur $S(X)$. Posons

$$
H(a, t, s)=(a, t) \quad \text { si }(a, t, s) \in S(X) \times I \times I .
$$

Pour $(a, t) \in M(\mathfrak{U}) \times I$ et $0 \leq s \leq 1 / 2$, posons

$$
H(a, t, s)= \begin{cases}(k(a, 2 s \lambda(\varrho(a))), t) & \text { si } a \in M(\bar{W}), \\ (a, t) & \text { si } a \in M(X \backslash V) .\end{cases}
$$

Cette définition a un sens car $k(a, s)=a$ si $a \in S(Q)$ et, si $a \in M(\bar{W}) \cap$ $M(X \backslash V)=M(\bar{W} \backslash V)$, alors $\lambda(\varrho(a))=0$, donc $k(a, 2 s \lambda(\varrho(a)))=k(a, 0)=$ $a$. Notant $h(a)$ la projection (indépendante de $t$ ) de $H(a, t, 1)$ sur $M(X)$, posons, pour $a \in M(\mathfrak{U})$ et $1 / 2 \leq s \leq 1$,

$$
H(a, t, s)=\left(k^{\prime}(h(a), 2 s-1), t\right),
$$

ce qui est compatible avec les définitions précédentes. La fonction $H$ est continue car sa restriction à chacun des fermés $S(X) \times I \times I, M(\bar{W}) \times I \times$ $[0,1 / 2], M(X \backslash V) \times I \times[0,1 / 2]$ et $M(\mathfrak{U}) \times I \times[1 / 2,1]$ l'est. Les conditions (i) et (ii) sont évidemment vérifiées, ainsi que (iv) car $k^{\prime}(M(X) \times\{1\}) \subset S(X)$. Si $a \in M(A)$, alors $\lambda(\varrho(a))=1$, donc, pour $0 \leq s \leq 1 / 2, H(a, t, s)=$ 
$(k(a, 2 s), t) \in M(Q) \times I$; en outre, $h(a)=k(a, 1) \in S(X)$, donc $H(a, t, s)=$ $H(a, t, 1 / 2)$ pour $1 / 2 \leq s \leq 1$, d'où (iii).

Les conditions (i)-(iv) entraînent que nous pouvons définir une fonction continue

$$
\begin{aligned}
\bar{H}:[(S(X) \cup M(\mathfrak{U})) \times I \times\{0\}] & \\
& \cup[((S(X) \cup M(A)) \times I \cup(M(\mathfrak{U}) \times\{0,1\})) \times I] \rightarrow K
\end{aligned}
$$

par

$$
\bar{H}(a, t, s)=F(H(a, t, 1-s)) .
$$

D'après (i), $\bar{H}(a, t, 1)=F(a, t)$ pour $(a, t) \in((S(X) \cup M(A)) \times I) \cup$ $(M(\mathfrak{U}) \times[0,1])$. Le lemme 7 , appliqué à l'inclusion

$$
((S(X) \cup M(A)) \times I) \cup(M(\mathfrak{U}) \times\{0,1\}) \hookrightarrow(S(X) \cup M(\mathfrak{U})) \times I,
$$

entraîne alors que $F \mid((S(X) \cup M(A)) \times I) \cup(M(\mathfrak{U}) \times\{0,1\})$ se prolonge en une fonction continue $\bar{F}:(S(X) \cup M(\mathfrak{U})) \times I \rightarrow K$. Nous pouvons prolonger $\bar{F}$ à $((S(X) \cup M(\mathfrak{U})) \times I) \cup(M(X) \times\{0,1\})$ en posant $\bar{F}(a, t)=$ $F(a, t)$ si $(a, t) \in M(X) \times\{0,1\}$, et le lemme 5 permet alors de trouver un prolongement continu $G$ de $\bar{F}$ à $M(X) \times I$; c'est la fonction cherchée.

Dans la section suivante, nous appliquerons les lemmes 9 et 10 au cas où $K=S(U), U$ ouvert de $X$, ce qui est légitime d'après le lemme 8 .

4. Démonstration du théorème. Comme il est indiqué dans l'introduction, il suffit de construire une fonction continue $\varphi: X \times] 0,1] \rightarrow S(X)$ et une homotopie $\Phi: X \times I \rightarrow X$ vérifiant $\Phi(x, 0)=x$ et $\Phi(x, t)=\pi \circ \varphi(x, t)$ pour $t>0$.

Supposons la distance de $X$ bornée par 1. Partant de $A_{0}=\{0\}$ et $\mathfrak{U}_{0}=$ $\{X\}$, construisons par récurrence des recouvrements ouverts localement finis $\mathfrak{U}_{n}=\left\{U_{\alpha} \mid \alpha \in A_{n}\right\}$ de $X$ vérifiant, pour $n \geq 0$,

(1) $\operatorname{diam}\left(U_{\alpha}\right) \leq 2^{-n} \quad \forall \alpha \in A_{n}$,

(2) $\overline{\mathfrak{U}}_{n+1}$ est plus fin que $\mathfrak{U}_{n}$,

(3) le nerf $N\left(\overline{\mathfrak{U}}_{n}\right)$ est localement de dimension finie.

La possibilité de trouver ces recouvrements découle de la paracompacité de $X$ et d'un résultat de C. H. Dowker ([4], lemme 3.3). Pour $\alpha \in A_{n}, n \geq 1$, choisissons $\lambda(\alpha) \in A_{n-1}$ tel que $U_{\lambda(\alpha)}$ contienne $\bar{U}_{\alpha}$.

Pour $\alpha \in A_{n}, n \geq 1$ et $p \geq 0$, prenons des ouverts $U_{\alpha}^{p}$ et des fermés $F_{\alpha}^{p}$ vérifiant

$$
\bar{U}_{\alpha} \subset U_{\alpha}^{p+1} \subset F_{\alpha}^{p+1} \subset U_{\alpha}^{p} \subset F_{\alpha}^{p} \subset U_{\lambda(\alpha)},
$$

la famille $\left\{F_{\alpha}^{0} \mid \alpha \in A_{n}\right\}$ est localement finie, 


$$
\begin{gathered}
\text { pour } \alpha_{0}, \ldots, \alpha_{i} \text { dans } A_{n}, F_{\alpha_{0}}^{0} \cap \ldots \cap F_{\alpha_{i}}^{0} \neq \emptyset \text { si, et seulement si, } \\
\bar{U}_{\alpha_{0}} \cap \ldots \cap \bar{U}_{\alpha_{i}} \neq \emptyset .
\end{gathered}
$$

(L'existence d'ensembles $F_{\alpha}^{p}$ et $U_{\alpha}^{p}$ vérifiant ces conditions résulte de la proposition 1.9, p. 60 de [11]).

Si $\sigma=\left\{\alpha_{0}, \ldots, \alpha_{i}\right\}$ est un simplexe de $N\left(\overline{\mathfrak{U}}_{n}\right)$ et $p$ un entier $\geq 0$, nous poserons $U_{\sigma}^{p}=U_{\alpha_{0}}^{p} \cap \ldots \cap U_{\alpha_{i}}^{p}$ et $F_{\sigma}^{p}=F_{\alpha_{0}}^{p} \cap \ldots \cap F_{\alpha_{i}}^{p}$; nous noterons $\lambda(\sigma)=\left\langle\lambda\left(\alpha_{0}\right), \ldots, \lambda\left(\alpha_{i}\right)\right\rangle$, qui est un simplexe de $N\left(\mathfrak{U}_{n-1}\right)$ (éventuellement de dimension inférieure à celle de $\sigma)$. Quand cela a un sens, nous écrirons $\lambda^{3}(\alpha)=\lambda \circ \lambda \circ \lambda(\alpha)$ et $\lambda^{3}(\sigma)=\lambda \circ \lambda \circ \lambda(\sigma)\left(\alpha \in A_{n}\right.$ et $\sigma$ simplexe de $\left.N\left(\overline{\mathfrak{U}}_{n}\right)\right)$. Pour tout simplexe $\sigma$ de $N\left(\overline{\mathfrak{U}}_{n}\right)$, posons

$$
\mu(\sigma)=\sup \{\operatorname{dim} \tau-\operatorname{dim} \sigma\}
$$

la borne supérieure étant prise sur tous les simplexes $\tau$ dont $\sigma$ est face. D'après $(3), \mu(\sigma)$ est un entier $\geq 0$. Si $\sigma$ est une face propre de $\tau$, alors $\mu(\sigma)>\mu(\tau)$.

Affirmation 1. Pour tout $n \geq 1$, il existe une fonction continue $f_{n}$ : $M(X) \rightarrow S(X)$ vérifiant

(i) $f_{n}(a)=a \forall a \in S(X)$,

(ii) $f_{n}\left(M\left(\bar{U}_{\alpha}\right)\right) \subset S\left(U_{\lambda(\alpha)}\right) \forall \alpha \in A_{n}$.

Fixons $n$. Nous allons d'abord construire, pour tout simplexe $\sigma$ de $N\left(\overline{\mathfrak{U}}_{n}\right)$, une fonction continue $f_{\sigma}: M\left(F_{\sigma}^{\mu(\sigma)}\right) \rightarrow S\left(U_{\lambda(\sigma)}\right)$ de façon que

$$
f_{\sigma}(a)=a \forall a \in S\left(F_{\sigma}^{\mu(\sigma)}\right),
$$

(8) $\quad$ si $\sigma$ est face de $\tau$, alors $f_{\sigma}\left|M\left(F_{\tau}^{\mu(\sigma)}\right)=f_{\tau}\right| M\left(F_{\tau}^{\mu(\sigma)}\right)$.

Si $\mu(\sigma)=0$, la condition (8) est vide, et l'on peut prendre pour $f_{\sigma}$ la restriction d'une rétraction de $M\left(U_{\lambda(\sigma)}\right)$ sur $S\left(U_{\lambda(\sigma)}\right)$, qui existe d'après le lemme 2. Soit $k \geq 1$ et supposons $f_{\tau}$ définie lorsque $\mu(\tau)<k$. Fixons un simplexe $\sigma$ avec $\mu(\sigma)=k$. Soit $\mathfrak{D}_{\sigma}$ l'ensemble des simplexes de $N\left(\overline{\mathfrak{U}}_{n}\right)$ dont $\sigma$ est une face propre, et soit $\mathfrak{F}_{\sigma}=\left\{F_{\tau}^{k-1} \mid \tau \in \mathfrak{D}_{\sigma}\right\}$. Si $\tau$ et $\tau^{\prime}$ sont deux éléments de $\mathfrak{D}_{\sigma}$ tels que $F_{\tau}^{k-1} \cap F_{\tau^{\prime}}^{k-1} \neq \emptyset$, alors, d'après (4) et (6), les sommets des simplexes $\tau$ et $\tau^{\prime}$ déterminent un simplexe $\bar{\tau}$ de $N\left(\overline{\mathfrak{U}}_{n}\right)$ pour lequel $F_{\tau}^{k-1} \cap F_{\tau^{\prime}}^{k-1}=F_{\bar{\tau}}^{k-1}$, d'où $M\left(F_{\tau}^{k-1}\right) \cap M\left(F_{\tau^{\prime}}^{k-1}\right)=M\left(F_{\bar{\tau}}^{k-1}\right)$. En utilisant (8), on constate que $f_{\tau}\left|M\left(F_{\bar{\tau}}^{k-1}\right)=f_{\tau^{\prime}}\right| M\left(F_{\bar{\tau}}^{k-1}\right)$, donc nous pouvons définir une fonction $g_{\sigma}: S\left(F_{\sigma}^{k-1}\right) \cup M\left(\mathfrak{F}_{\sigma}\right) \rightarrow S(X)$ par

$$
\begin{gathered}
g_{\sigma}(a)=a \quad \text { si } a \in S\left(F_{\sigma}^{k-1}\right), \\
g_{\sigma}\left|M\left(F_{\tau}^{k-1}\right)=f_{\tau}\right| M\left(F_{\tau}^{k-1}\right) \quad \forall \tau \in \mathfrak{D}_{\sigma} .
\end{gathered}
$$

Comme $f_{\tau}\left(M\left(F_{\tau}^{k-1}\right)\right) \subset S\left(U_{\lambda(\tau)}\right), g_{\sigma}$ est à valeurs dans $S\left(U_{\lambda(\sigma)}\right)$. La restriction de $g_{\sigma}$ à chacun des fermés $S\left(F_{\sigma}^{k-1}\right)$ et $M\left(F_{\tau}^{k-1}\right), \tau \in \mathfrak{D}_{\sigma}$, est 
continue; il résulte de (5) que cette famille est localement finie dans $M(X)$, donc $g_{\sigma}$ est continue.

Soit $\mathfrak{Q}_{\sigma}=\left\{U_{\tau}^{k-1} \mid \tau \in \mathfrak{D}_{\sigma}\right\}$, et soit $Q$ la réunion des éléments de $\mathfrak{Q}_{\sigma}$; $Q$ est un ouvert contenu dans $U_{\sigma}^{k-1}$. Le lemme 4 permet de trouver une fonction continue $g_{\sigma}^{\prime}: M(Q) \rightarrow S\left(U_{\lambda(\sigma)}\right)$ égale à $g_{\sigma}$ sur $S(Q) \cup M\left(\mathfrak{Q}_{\sigma}\right)$. D'après (5), la famille des fermés $F_{\tau}^{k}$ avec $\tau \in \mathfrak{D}_{\sigma}$ est localement finie; sa réunion $P$ est donc fermée, et est contenue dans $Q$ d'après (4). Appliquant le lemme 9 , en y remplaçant $X$ par $U_{\sigma}^{k-1}$ et $A$ par $P$, nous pouvons trouver une fonction continue $g_{\sigma}^{\prime \prime}: M\left(U_{\sigma}^{k-1}\right) \rightarrow S\left(U_{\lambda(\sigma)}\right)$ telle que $g_{\sigma}^{\prime \prime}\left|M(P)=g_{\sigma}^{\prime}\right| M(P)$ et $g_{\sigma}^{\prime \prime}(a)=a$ pour $a \in S\left(U_{\sigma}^{k-1}\right)$. Soit $f_{\sigma}$ la restriction de $g_{\sigma}^{\prime \prime}$ à $M\left(F_{\sigma}^{k}\right)$. La condition (7) est vérifiée par construction, et (8) résulte du fait que si $\tau \in \mathfrak{D}_{\sigma}$, alors $F_{\tau}^{k} \subset P$, et $g_{\sigma}^{\prime \prime}, g_{\sigma}^{\prime}, g_{\sigma}$ et $f_{\tau}$ coïncident sur $M\left(F_{\tau}^{k}\right)$.

Notant simplement $\alpha$ le 0 -simplexe associé à un élément $\bar{U}_{\alpha}$ de $\overline{\mathfrak{U}}_{n}$ (nous supposons les $U_{\alpha}$ non vides), et $f_{\alpha}$ la fonction associée ci-dessus à ce 0 simplexe, il résulte de (8), appliqué aux 1-simplexes $\left\langle\alpha, \alpha^{\prime}\right\rangle$ de $N\left(\overline{\mathfrak{U}}_{n}\right)$, que $f_{\alpha}\left|M\left(\bar{U}_{\alpha}\right) \cap M\left(\bar{U}_{\alpha^{\prime}}\right)=f_{\alpha^{\prime}}\right| M\left(\bar{U}_{\alpha}\right) \cap M\left(\bar{U}_{\alpha^{\prime}}\right)$. D'après (7), nous pouvons donc définir une fonction $f^{\prime}: S(X) \cup M\left(\overline{\mathfrak{U}}_{n}\right) \rightarrow S(X)$ par

$$
\begin{gathered}
f^{\prime}(a)=a \quad \forall a \in S(X), \\
f^{\prime}\left|M\left(\bar{U}_{\alpha}\right)=f_{\alpha}\right| M\left(\bar{U}_{\alpha}\right) \quad \forall \alpha \in A_{n} .
\end{gathered}
$$

La continuité de $f^{\prime}$ résulte encore de la finitude locale de la famille de fermés $M\left(\bar{U}_{\alpha}\right), \alpha \in A_{n}$. Le lemme 4 permet alors de prolonger $f^{\prime}$ en une fonction continue $f_{n}: M(X) \rightarrow S(X)$. La condition (i) est trivialement vérifiée, et (ii) résulte de ce que $f^{\prime}\left|M\left(\bar{U}_{\alpha}\right)=f_{\alpha}\right| M\left(\bar{U}_{\alpha}\right)$.

Affirmation 2. Pour tout $n \geq 2$, il existe une homotopie $h_{n}: M(X) \times I$ $\rightarrow S(X)$ vérifiant

(i) $h_{n}(a, t)=a \quad \forall(a, t) \in S(X) \times I$,

(ii) $h_{n}(y, 0)=f_{n}(y), h_{n}(y, 1)=f_{n-1}(y) \quad \forall y \in M(X)$,

(iii) $h_{n}\left(M\left(\bar{U}_{\alpha}\right) \times I\right) \subset S\left(U_{\lambda^{3}(\alpha)}\right) \forall \alpha \in A_{n+1}$.

La démonstration est parallèle à celle de l'affirmation 1 , mais utilise les lemmes 5 et 10 au lieu des lemmes 4 et 9 . Nous commençons par construire, par récurrence sur $\mu(\sigma)$, pour tout simplexe $\sigma$ de $N\left(\overline{\mathfrak{U}}_{n+1}\right)$, une homotopie $h_{\sigma}: M\left(F_{\sigma}^{\mu(\sigma)}\right) \times I \rightarrow S\left(U_{\lambda^{3}(\sigma)}\right)$ vérifiant

$$
\begin{aligned}
& h_{\sigma}(a, t)=a \forall(a, t) \in S\left(F_{\sigma}^{\mu(\sigma)}\right) \times I, \\
& h_{\sigma}(y, 0)=f_{n}(y), h_{\sigma}(y, 1)=f_{n-1}(y) \forall y \in M\left(F_{\sigma}^{\mu(\sigma)}\right), \\
& \text { si } \sigma \text { est face de } \tau, \text { alors } h_{\sigma}\left|M\left(F_{\tau}^{\mu(\sigma)}\right) \times I=h_{\tau}\right| M\left(F_{\tau}^{\mu(\sigma)}\right) \times I .
\end{aligned}
$$

Si $\mu(\sigma)=0$, on peut, en remarquant que, d'après (ii) de l'affirmation 1 , $f_{n}$ et $f_{n-1}$ envoient $M\left(U_{\lambda(\sigma)}\right)$ dans $S\left(U_{\lambda^{3}(\sigma)}\right)$, prendre pour $h_{\sigma}$ la restriction d'une homotopie $h$ de $M\left(U_{\lambda(\sigma)}\right) \times I$ dans $S\left(U_{\lambda^{3}(\sigma)}\right)$ vérifiant $h(a, t)=a$ si 
$(a, t) \in S\left(U_{\lambda(\sigma)}\right) \times I, h(y, 0)=f_{n}(y)$ et $h(y, 1)=f_{n-1}(y) \forall y \in M\left(U_{\lambda(\sigma)}\right)$ (l'existence de $h$ est garantie par le lemme 10, avec $X=U_{\lambda(\sigma)}, A=Q=\emptyset$ et $\left.K=S\left(U_{\lambda^{3}(\sigma)}\right)\right)$. Supposant $\mu(\sigma)=k>0$ et $h_{\tau}$ construite pour $\mu(\tau)<k$, définissons $\mathfrak{D}_{\sigma}, \mathfrak{F}_{\sigma}, \mathfrak{Q}_{\sigma}, Q$ et $P$ comme précédemment (en changeant $n$ en $n+1)$. Les $h_{\tau}, \tau \in \mathfrak{D}_{\sigma}$, permettent de définir une fonction continue

$$
k_{\sigma}:\left(\left(S\left(F_{\sigma}^{k-1}\right) \cup M\left(\mathfrak{F}_{\sigma}\right)\right) \times I\right) \cup\left(M\left(F_{\sigma}^{k-1}\right) \times\{0,1\}\right) \rightarrow S\left(U_{\lambda^{3}(\sigma)}\right)
$$

par

$$
\begin{gathered}
k_{\sigma}(a, t)=a \quad \text { si }(a, t) \in S\left(F_{\sigma}^{k-1}\right) \times I, \\
k_{\sigma}\left|M\left(F_{\tau}^{k-1}\right) \times I=h_{\tau}\right| M\left(F_{\tau}^{k-1}\right) \times I \quad \forall \tau \in \mathfrak{D}_{\sigma}, \\
k_{\sigma}(y, 0)=f_{n}(y), \quad k_{\sigma}(y, 1)=f_{n-1}(y) \quad \forall y \in M\left(F_{\sigma}^{k-1}\right) .
\end{gathered}
$$

Le lemme 5 permet de trouver une fonction continue $k_{\sigma}^{\prime}: M(Q) \times I \rightarrow$ $S\left(U_{\lambda^{3}(\sigma)}\right)$ égale à $k_{\sigma} \operatorname{sur}\left(\left(S(Q) \cup M\left(\mathfrak{Q}_{\sigma}\right)\right) \times I\right) \cup(M(Q) \times\{0,1\})$, puis le lemme 10 de trouver une homotopie $k_{\sigma}^{\prime \prime}: M\left(U_{\sigma}^{k-1}\right) \times I \rightarrow S\left(U_{\lambda^{3}(\sigma)}\right)$ vérifiant

$$
\begin{gathered}
k_{\sigma}^{\prime \prime}(a, t)=a \quad \text { si }(a, t) \in S\left(U_{\sigma}^{k-1}\right) \times I, \\
k_{\sigma}^{\prime \prime}(y, 0)=f_{n}(y), \quad k_{\sigma}^{\prime \prime}(y, 1)=f_{n-1}(y) \quad \forall y \in M\left(U_{\sigma}^{k-1}\right), \\
k_{\sigma}^{\prime \prime}\left|M(P) \times I=k_{\sigma}^{\prime}\right| M(P) \times I .
\end{gathered}
$$

On peut alors prendre pour $h_{\sigma}$ la restriction de $k_{\sigma}^{\prime \prime}$ à $M\left(F_{\sigma}^{k}\right) \times I$. Notant $h_{\alpha}$ la fonction ainsi associée à un 0-simplexe $\alpha$ de $N\left(\overline{\mathfrak{U}}_{n+1}\right)$, définissons $h^{\prime}:\left(\left(S(X) \cup M\left(\overline{\mathfrak{U}}_{n+1}\right)\right) \times I\right) \cup(M(X) \times\{0,1\}) \rightarrow S(X)$ par

$$
\begin{gathered}
h^{\prime}(a, t)=a \quad \forall(a, t) \in S(X), \\
h^{\prime}\left|M\left(\bar{U}_{\alpha}\right) \times I=h_{\alpha}\right| M\left(\bar{U}_{\alpha}\right) \times I \quad \forall \alpha \in A_{n+1}, \\
h^{\prime}(y, 0)=f_{n}(y), \quad h^{\prime}(y, 1)=f_{n-1}(y) \quad \forall y \in M(X) .
\end{gathered}
$$

Le lemme 5 permet de prolonger $h^{\prime}$ en une homotopie $h_{n}: M(X) \times I \rightarrow$ $S(X)$ qui a les propriétés souhaitées.

Définissons alors $\varphi: X \times] 0,1] \rightarrow S(X)$ par

$$
\varphi(x, t)=h_{n+1}\left(x, 2^{n}\left(t-2^{-n}\right)\right) \quad \text { pour } 2^{-n} \leq t \leq 2^{-n+1}, n \geq 1 .
$$

Puisque $h_{n+2}(x, 1)=f_{n+1}(x)=h_{n+1}(x, 0)$, cette définition a un sens; les $h_{n}$ étant continues, $\varphi$ aussi. Posons ensuite

$$
\Phi(x, t)= \begin{cases}x & \text { si } t=0 \\ \pi \circ \varphi(x, t) & \text { si } 0<t \leq 1 .\end{cases}
$$

La fonction $\Phi$ est continue sur $X \times] 0,1]$. Remarquons que, d'après (iii) de l'affirmation 2, si $\alpha \in A_{n+2}$ est tel que $U_{\alpha}$ contienne $x$, alors, pour $2^{-n} \leq t \leq 2^{-n+1}, U_{\lambda^{3}(\alpha)}$ contient $x$ et $\Phi(x, t)$, d'où, d'après (1), puisque $\lambda^{3}(\alpha) \in A_{n-1}$,

$$
d(x, \Phi(x, t)) \leq 2^{-n+1} \quad \text { pour } 2^{-n} \leq t \leq 2^{-n+1}, n \geq 1 .
$$

La continuité de $\Phi$ aux points $(x, 0)$ en résulte, d'où le théorème. 


\section{Bibliographie}

[1] C. J. R. Borges, On stratifiable spaces, Pacific J. Math. 17 (1966), 1-16.

[2] R. Cauty, Convexité topologique et prolongement des fonctions continues, Compositio Math. 27 (1973), 233-271.

[3] T. tom Dieck, K. H. Kamps und D. Puppe, Homotopietheorie, Lecture Notes in Math. 157, Springer, Berlin, 1970.

[4] C. H. Dowker, Mapping theorems for non-compact spaces, Amer. J. Math. 69 (1947), 200-242.

[5] R. Engelking, General Topology, PWN, Warszawa, 1977.

[6] R. Geoghegan, Conjecture 6, in: Proc. Internat. Conf. on Geometric Topology, Warszawa, 1978, Presented Problems, PWN, Warszawa, 1980, p. 463.

[7] _, Open problems in infinite-dimensional topology, Topology Proc. 4 (1979), 287338.

[8] J. B. Giever, On the equivalence of two singular homology theories, Ann. of Math. 51 (1950), 178-191.

[9] S. T. Hu, Theory of Retracts, Wayne State University Press, Detroit, 1965.

[10] G. Kozlowski, Images of ANR's, manuscrit non publié.

[11] A. R. Pears, Dimension Theory of General Spaces, Cambridge University Press, Cambridge, 1975.

[12] E. H. Spanier, Algebraic Topology, McGraw-Hill, New York, 1966.

[13] A. Strøm, Note on cofibrations II, Math. Scand. 22 (1968), 130-142.

[14] J. E. West, Open problems in infinite dimensional topology, in: Open Problems in Topology, J. van Mill and G. M. Reed (eds.), Elsevier, 1990, 524-597.

22, RUE JOUVENET

F-75016 PARIS, FRANCE

Received 3 March 1992;

in revised form 15 April 1993 